\title{
Origin of Ly $\alpha$ absorption in nearby starbursts and implications for other galaxies ${ }^{\star}$
}

\author{
H. Atek ${ }^{1}$, D. Schaerer ${ }^{2,3}$, and D. Kunth ${ }^{1}$ \\ 1 Institut d'Astrophysique de Paris (IAP), 98bis boulevard Arago, 75014 Paris, France \\ e-mail: atek@iap.fr \\ 2 Observatoire de Genève, Université de Genève, 51 Ch. des Maillettes, 1290 Sauverny, Switzerland \\ ${ }^{3}$ Laboratoire d'Astrophysique de Toulouse-Tarbes, Université de Toulouse, CNRS, 14 Avenue E. Belin, 31400 Toulouse, France
}

Received 16 February 2009 / Accepted 6 May 2009

\begin{abstract}
Context. Despite the privileged position that Lyman- $\alpha(\operatorname{Ly} \alpha)$ emission line holds in the exploration of the distant universe and modern observational cosmology, the origin of the observed diversity of Ly $\alpha$ profiles still needs to be thoroughly explained. Observations of nearby star forming galaxies bring their batch of apparent contradictions between Ly $\alpha$ emission and their physical parameters, and call for a detailed understanding of the physical processes at work. One of the most metal-poor galaxies known, IZw 18, is of particular interest in this context.

Aims. We fit the Ly $\alpha$ spectrum of IZw 18 to understand the origin of the damped absorption profile and its spatial variations across the NW region, and establish a general picture of the physical parameters governing the Ly $\alpha$ strength and profile both in local and in high- $z$ galaxies.

Methods. We used a 3D Ly $\alpha$ radiation transfer code to model Hubble Space Telescope (HST) observations of IZw 18. Observational constraints of relevant parameters such as dust or HI column density were derived from previous studies and from the present analysis. Different geometrical configurations of the source and the neutral gas were explored.

Results. The integrated Ly $\alpha$ profile of NW region of IZw 18 is reproduced using the observed small amount of dust $(E(B-V) \approx 0.05)$ and a spherical $\mathrm{HI}$ shell with $N_{\mathrm{HI}}=6.5 \times 10^{21} \mathrm{~cm}^{-2}$. Such a high column density makes it possible to transform a strong Ly $\alpha$ emission $\left(E W_{\mathrm{Ly} \alpha}=60 \AA\right)$ into a damped absorption even with low extinction. When a slab geometry is applied and a given line of sight is chosen, the Ly $\alpha$ profile can be successfully reproduced with no dust at all and $N_{\mathrm{HI}}=3 \times 10^{21} \mathrm{~cm}^{-2}$. The spatial variations of the profile shape are naturally explained by radiation transfer effects, i.e. by scattering of Ly $\alpha$ photons, when the observed surface brightness profile of the source is taken into account. In the case of outflowing inter stellar medium (ISM), as commonly observed in Lyman break galaxies (LBGs), a high $N_{\mathrm{HI}}$ and dust content are required to observe Ly $\alpha$ in absorption. For nearly static neutral gas as observed in IZw 18 and other low-luminosity galaxies, only a small amount of dust is required provided a sufficiently high $N_{\mathrm{HI}}$ covers the galaxy. We also show how geometrical and apertures effects affect the Ly $\alpha$ profile.
\end{abstract}

Key words. galaxies: starburst - galaxies: ISM - ultraviolet: galaxies - radiative transfer - galaxies: individual: IZw 18

\section{Introduction}

The detection of high-redshift ( $z$ ) galaxies has become, through the last decade, a routine fact, although the discovery of primeval galaxies that are forming their first stars still remains a challenge. Depending on the selection techniques, mainly two classes of galaxies are found: Lyman break galaxies (LBGs) selected by their UV continuum break, and Lyman-alpha emitters (LAEs) selected upon their Ly $\alpha$ emission line. The situation was, however far different before this successful era. Partridge \& Peebles (1967) were the first to estimate that young distant galaxies should be detectable through a strong Ly $\alpha$ emission. Nevertheless, it took nearly thirty years until such populations could be found at $z \sim 2-7$, thanks in particular to instruments with large fields of view (FOV) and 4-8 m class telescopes (see e.g. Hu et al. 1998; Kudritzki et al. 2000; Malhotra \& Rhoads 2002; Ajiki et al. 2003; Taniguchi et al. 2005; Shimasaku et al. 2006; Kashikawa et al. 2006; Tapken et al. 2006; Gronwall et al. 2007; Ouchi et al. 2008; Nilsson et al. 2009). Only recently, has GALEX provided a comparable survey for the first time at low

* Based on observations made with the Hubble Space Telescope obtained from the ESO/ST-ECF Science Archive Facility. redshift $(z \sim 0.2-0.35$, Deharveng et al. 2008), thanks to its wide FOV and spectroscopic capabilities in the UV.

Earlier studies of nearby galaxies using mostly the UV capabilities of IUE and HST recognized quickly that Ly $\alpha$ emission was fainter than naively expected from recombination theory and that the $\operatorname{Ly} \alpha$ line showed a great diversity of profiles from absorption to emission (e.g. Meier \& Terlevich 1981; Hartmann et al. 1984; Deharveng et al. 1985; Hartmann et al. 1988; Terlevich et al. 1993; Lequeux et al. 1995; Thuan et al. 1997; Thuan \& Izotov 1997; Kunth et al. 1998). Later, HST has allowed mapping the spatial distribution of $\operatorname{Ly} \alpha$ emission and absorption, of the stellar sources, nebular emission, and dust (see Kunth et al. 2003; Mas-Hesse et al. 2003; Hayes et al. 2005, 2008; Atek et al. 2008). Despite all this information, no clear picture has yet emerged to consistently explain the Ly $\alpha$ and related observations in nearby starbursts.

Indeed, it is now well known, both theoretically and empirically, that different physical processes affect the Ly $\alpha$ intensity, profile shape, and "visibility" (i.e. detection frequency among starbursts $)^{1}$ : destruction of $\operatorname{Ly} \alpha$ photons by dust

\footnotetext{
1 See Schaerer (2007) for an overview.
} 
(cf. Neufeld 1990; Charlot \& Fall 1993), velocity fields in the ISM (Kunth et al. 1998; Lequeux et al. 1995), an inhomogeneous ISM (Neufeld 1991; Giavalisco et al. 1996; Hansen \& Peng Oh 2006), underlying stellar absorption (Valls-Gabaud 1993), and star-formation duty cycles (Valls-Gabaud 1993; Malhotra \& Rhoads 2002).

Also a "unifying" scenario to explain the observed diversity of Ly $\alpha$ profiles in terms of an evolutionary sequence of starburstdriven super-shells/superwind was presented by Tenorio-Tagle et al. (1999) and has since been compared with local starburst observations by Mas-Hesse et al. (2003). For distant galaxies, Schaerer \& Verhamme (2008) and Verhamme et al. (2008) have recently shown - using radiation transfer models and empirical constraints - that Ly $\alpha$ line profiles of high- $z$ LBGs and LAEs can be reproduced well and that the diversity of Ly $\alpha$ from emission to absorption stems mainly from an increase in the dust content and the HI column density. Despite this progress, a global picture identifying the main processes and explaining this diversity also in a quantitative manner is still missing for $\operatorname{Ly} \alpha$ in local/nearby galaxies. Furthermore, differences between the high and low redshift samples - if any - must be understood. We here provide a first step towards these goals by examining and modelling one of the most metal-poor star-forming galaxies in the local Universe, IZw 18 , and by putting it into context.

Since its discovery by Zwicky (1966), IZw 18 has been studied extensively, and it remains one of the most metal-poor galaxies known today (Skillman \& Kennicutt 1993; Izotov \& Thuan 1999). Its main HII region (called the NW region, cf. Fig. 1) showing strong optical emission lines is clearly a site of very recent ( $<10 \mathrm{Myr}$ ) and/or ongoing massive star formation (cf. Hunter \& Thronson 1995; de Mello et al. 1998; Brown et al. 2002). Therefore the finding of a broad damped Ly $\alpha$ absorption line by Kunth et al. $(1994,1998)$ came as a surprise, where strong emission was predicted, given the strong optical $\mathrm{H}$ recombination lines and the low dust content expected for such low metallicities (cf. Kunth et al. 1994; Terlevich et al. 1993). Observations of SBS 0335-052, nearly as metal-poor as IZw 18, showed later a similarly broad profile (Thuan \& Izotov 1997). However, since compared to IZw 18, SBS 0335-052 has a higher extinction and is now known to harbor more dust both in absolute terms (dust mass) and in relative terms $\left(L_{\mathrm{IR}} / L_{\mathrm{UV}}\right)$ (Thuan et al. 1999; Houck et al. 2004; Wu et al. 2007; Engelbracht et al. 2008), it is more challenging to explain Ly $\alpha$ absorption in IZw 18 than in SBS 0335-052. For these reasons IZw 18 represents an ideal test case to understand how strong intrinsic Ly $\alpha$ emission is transformed to the observed broad Ly $\alpha$ absorption, in a dust-poor (but not dust-free), very metal-poor galaxy.

Kunth et al. (1994, 1998) suggested that both dust absorption and multiple scattering of $\operatorname{Ly} \alpha$ photons out of their narrow $\left(2.0^{\prime \prime} \times 2.0^{\prime \prime}\right)$ GHRS/HST aperture offer the most natural explanation for the observed weakness of Ly $\alpha$ in IZw 18. They also noted that all galaxies showing Ly $\alpha$ absorption (4/8 in their small sample) showed nearly static neutral gas, which must increase the mean free path of Ly $\alpha$ photons. However, the IUE spectrum of IZw 18 taken with an entrance hole of $20^{\prime \prime} \times 10^{\prime \prime}$ shows basically the same profile, indicating that no significant amount of Ly $\alpha$ emission is recovered at least over 5-10 times larger scales. In any case, whether quantitatively these explanations are viable remains to be seen. This is one of the concrete goals of the present paper.

To address the above questions we use the most recent observations of IZw 18 and our state-of-the-art 3D Ly $\alpha$ and UV continuum radiation transfer code MCLya (Verhamme et al. 2006). This will in particular allow us to carefully examine in a
Table 1. HST observations of IZw 18.

\begin{tabular}{llcccc}
\hline \hline Instrument & $\begin{array}{l}\text { Filter/ } \\
\text { Grating }\end{array}$ & Band & $\begin{array}{c}\text { Exposure } \\
\text { time [s] }\end{array}$ & $\begin{array}{c}\text { Proposal } \\
\text { ID }\end{array}$ & Ref. $^{a}$ \\
\hline STIS & $G 140 M$ & Ly $\alpha$ & 1764 & GO-8302 & 1 \\
STIS & $G 140 L$ & Ly $\alpha$ & $40360^{b}$ & GO-9054 & 2 \\
STIS & $F 25 S R F 2$ & FUV & 5331 & GO-9054 & 2 \\
STIS & $F 25 Q T Z$ & NUV & 5786 & GO-9054 & 2 \\
WFPC2 & $F 487 N$ & H $\beta$ & 2500 & GO-6536 & 3 \\
WFPC2 & $F 658 N$ & H $\alpha$ & 4600 & GO-5434 & 3 \\
WFPC2 & $F 450 W$ & $\mathrm{~B}$ & 4600 & GO-5434 & 3 \\
WFPC2 & $F 675 W$ & $\mathrm{R}$ & 2000 & GO-5434 & 3 \\
\hline
\end{tabular}

a References: (1) Mas-Hesse et al. (2003); (2) Brown et al. (2002); (3) Cannon et al. (2002).

${ }^{b}$ Total integration time in the 7 slit positions.

quantitative manner the possible explanations leading to Ly $\alpha$ absorption in IZw 18. Finally, we also discuss other nearby starbursts with $\operatorname{Ly} \alpha$ absorption, and place the local objects in a broader context.

Our paper is structured as follows. In Sect. 2 we describe the main observations from HST and other facilities and summarise the main observational constraints. In Sect. 3 we set out to explain the Ly $\alpha$ absorption in IZw 18, discussing our radiation transfer modelling tool and geometrical effects and presenting modeling results for different ISM geometries. Our results for IZw 18 are discussed and compared to other nearby and high- $z$ starbursts in Sect. 4. In Sect. 5 we summarise our main conclusions.

\section{Observations}

The main observational data used in this paper are summarized in Table 1. We present the instrument configuration, the exposure time and the reference of the program of observations. We describe in this section the method to determine the physical parameters of IZw 18 using the archival spectroscopic and imaging data. Other observational constraints are also taken from previous results in the literature.

\subsection{Spectroscopy}

We use archival spectroscopic observations obtained with the Space Telescope Imaging Spectrograph (STIS) onboard HST, under program GO-9054, by Brown et al. (2002). The G140L grating was used combined with the $52^{\prime \prime} \times 0.5^{\prime \prime}$ slit. IZw 18 was spatially covered with seven adjacent slit positions along its main axis (see Fig. 1). Standard calibrations were performed using the CALSTIS pipeline (Ver 2.26), and exposures (two) for each position are registered and co-added. In addition, data were corrected for geocoronal Ly $\alpha$ emission by fitting and subtracting the nearby background regions in individual spectra. This calibration and spectra extraction were performed with IRAF and IDL routines.

In Fig. 2 we show the spatial variations of the $\operatorname{Ly} \alpha$ profile across the NW region. Spectra were extracted from the seven adjacent positions of the STIS long slit covering the galaxy in the NE-SW axis, providing spatial information in two directions. Flux was then integrated in each slit along a 4 " aperture centered on the NW component of IZw 18. Finally, an integrated spectrum of the NW region was also constructed from these integrated slit spectra. The strength of the Ly $\alpha$ absorption in these spectra is quantified by its equivalent width and corresponding 


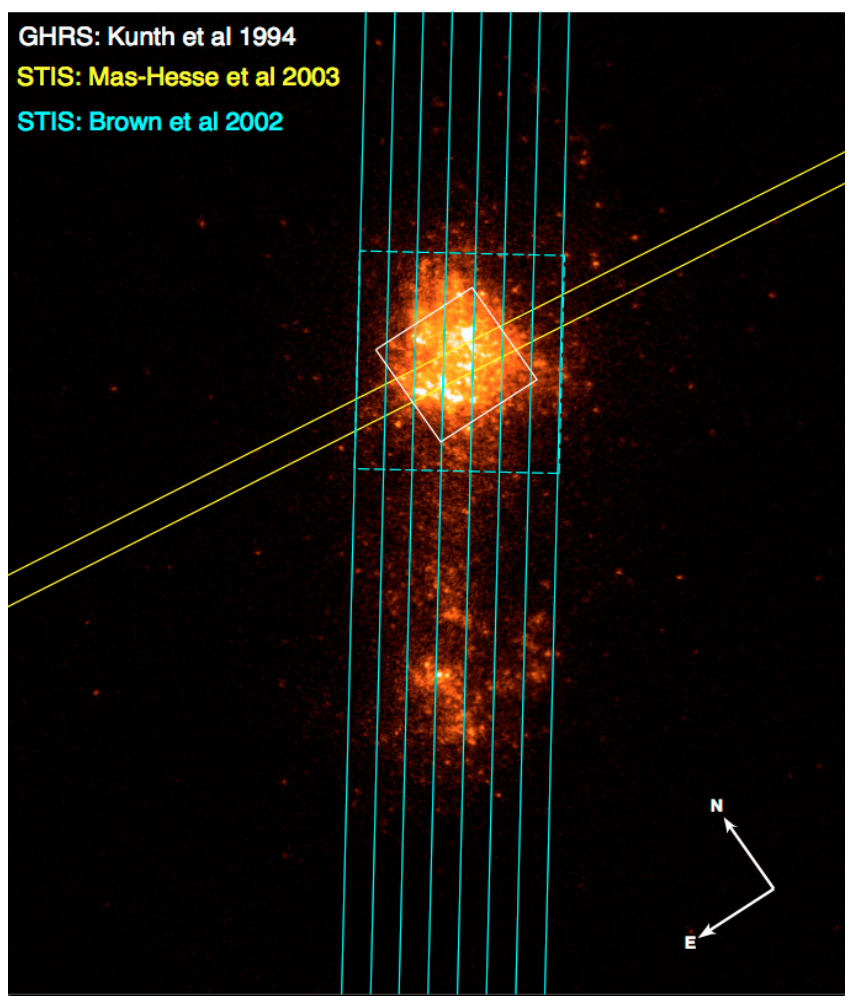

Fig. 1. FUV Image of IZw 18 showing the different observation apertures. The FOV is $16^{\prime \prime} \times 18^{\prime \prime}$. The first slit position is in the NE direction and the seventh toward the SW. The integration is performed within a region of 4 " along the spatial axis of the N-W region and is marked with dashed lines.

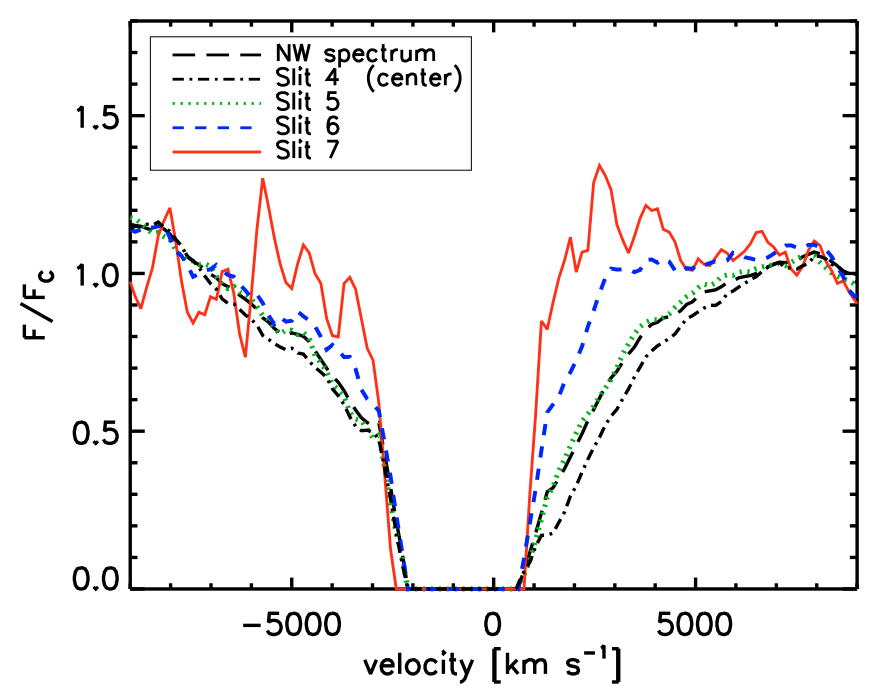

Fig. 2. STIS spectra of the NW region of IZw 18 taken at different locations (cf. Fig. 1). The slit positions 4 to 7 show the variation in the profile shape from the center to the edge of NW region. For comparison, the integrated spectrum in the seven slits over the center of NW region is overplotted. All spectra were normalized to the continuum value determined in Fig. 3. For the sake of clarity, a cut-off is applied on geocoronal emission residuals and spectra smoothed with a 3-pixel boxcar.

HI column density, $N_{\mathrm{HI}}$, determined assuming a Voigt profile and $b=20 \mathrm{~km} \mathrm{~s}^{-1}$ (cf. below). These values are reported in Table 2.

Earlier, Ly $\alpha$ observations of IZw 18 were obtained by Kunth et al. (1994) and later on by Kunth et al. (1998) using the Large Science Aperture (LSA, $2^{\prime \prime} \times 2^{\prime \prime}$ ) of GHRS onboard HST (see
Table 2. Spatial variations of Ly $\alpha$ properties in STIS slit positions.

\begin{tabular}{llcccc}
\hline \hline Slit & $\begin{array}{l}N_{\mathrm{HI}} \\
{\left[\mathrm{cm}^{-2}\right]}\end{array}$ & $\begin{array}{c}\sigma_{N_{\mathrm{H}}} \\
\left(\times 10^{21}\right)\end{array}$ & $\begin{array}{c}E W_{\text {Obs }} \\
{[\AA]}\end{array}$ & $\sigma_{E W}$ & $\begin{array}{c}f(1500 \AA) \\
{\left[\mathrm{erg} \mathrm{s}^{-1} \mathrm{~cm}^{-2}\right]}\end{array}$ \\
\hline 1 & $1.8 \times 10^{21}$ & 0.7 & -31 & 5 & $2.8 \times 10^{-16}$ \\
2 & $2.4 \times 10^{21}$ & 0.5 & -32 & 3 & $9.6 \times 10^{-16}$ \\
3 & $2.8 \times 10^{21}$ & 0.5 & -35 & 3 & $3.0 \times 10^{-15}$ \\
4 & $2.8 \times 10^{21}$ & 0.8 & -34 & 5 & $4.1 \times 10^{-15}$ \\
5 & $2.0 \times 10^{21}$ & 0.7 & -30 & 5 & $2.9 \times 10^{-15}$ \\
6 & $1.0 \times 10^{21}$ & 0.6 & -20 & 6 & $1.3 \times 10^{-15}$ \\
7 & $2.5 \times 10^{20}$ & 0.1 & -10 & 3 & $5.8 \times 10^{-16}$ \\
\hline NW & $2.1 \times 10^{21}$ & 0.7 & -31 & 5 & $1.3 \times 10^{-14}$ \\
MH03 & $2.2 \times 10^{21}$ & 0.7 & -30 & 4 & $2.6 \times 10^{-15}$ \\
\hline
\end{tabular}

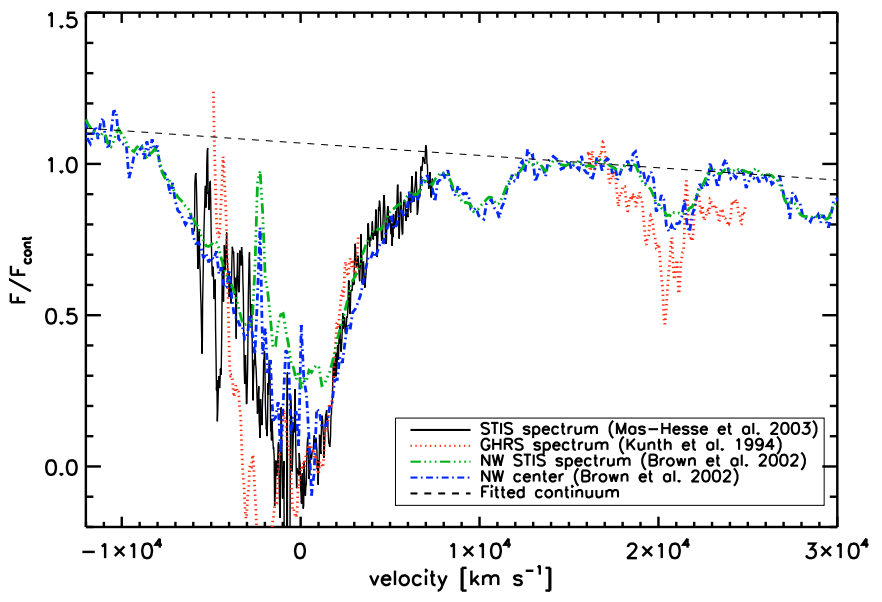

Fig. 3. IZw 18 spectroscopic data. The figure presents a compilation of spectroscopic informations available for IZw 18. Geocoronal Ly $\alpha$ emission has been subtracted from all the spectra. Dark solid line represents the best STIS spectrum around the Ly $\alpha$ absorption. The red dotted line is the GHRS spectrum, which covers a part of the absorption and a part of the UV continuum. The blue dot-dashed line shows the STIS spectrum with a large wavelength coverage ([1100-1750 ̊]]) extracted from the center of the NW region. The green long dot-dashed line is the result of an integration over all the slits in the NW region, previously shown in Fig. 2. It has been used to fit the UV continuum (black dashed line). All the spectra were then normalized to match the continuum value around $1280 \AA$ (i.e. at $v \sim 16000 \mathrm{~km} \mathrm{~s}^{-1}$ ). References and legend are given in the inset.

Fig. 1). Mas-Hesse et al. (2003, hereafter MH03) observed the galaxy again with better settings using STIS with G140M grating through a $52^{\prime \prime} \times 0.5^{\prime \prime}$ longslit, translating to a spectral resolution around $0.15 \AA$ ( $37 \mathrm{~km} \mathrm{~s}^{-1}$ at Ly $\alpha$ wavelength). The longer wavelength range of the STIS observations allows a better coverage of the Ly $\alpha$ absorption red wing, as compared to GHRS spectrum, and confirms the large damped Ly $\alpha$ absorption. In Fig. 3, we plot together the STIS and GHRS spectra. Because of differences in the instrument apertures, the spectra had to be matched. To fit and estimate the UV continuum we used the archival STIS observations that allows a broad wavelength coverage to include the absorption wings. All the spectra were then normalized to the value where the $\operatorname{Ly} \alpha$ red wing reaches this continuum $(\sim 1300 \AA)$. The different Ly $\alpha$ profiles obtained are in good agreement. A correct estimation of the continuum around Ly $\alpha$ is particularly important for the modeling of the Ly $\alpha$ spectral profile (see Sect. 3.2). 
Table 2 presents line measurements of the Ly $\alpha$ profile in the different slits. Columns (2) and (4) indicate the HI column density and the Ly $\alpha$ line equivalent width derived by fitting each Ly $\alpha$ absorption with a Voigt profile with $b=20 \mathrm{~km} \mathrm{~s}^{-1}$. The errors in Cols. (3) and (5) are determined from the lower and upper limits of the fits. The last column is the integrated flux in FUV (1500 ̊) image over the NW region and in simulated slits in order to match the aperture used for the extraction of STIS spectra. The same quantities are also given for integrated spectrum in the entire NW region and for the MH03 STIS spectrum.

Clearly, Ly $\alpha$ shows a broad absorption over the entire extent of the NW region. The width of the profile corresponds to an HI column density of $N_{\mathrm{HI}} \sim(0.3-3) \times 10^{21} \mathrm{~cm}^{-2}$, in agreement with earlier determinations $\left(N_{\mathrm{HI}} \sim(1.0-3.2) \times 10^{21} \mathrm{~cm}^{-2}\right.$ from UV observations by Kunth et al. (1998), although this method does not systematically give the true value of $N_{\mathrm{HI}}$, as we will see later on. Beyond the scale of the HII NW region $(\sim 250 \mathrm{pc})$, the UV-optical part of IZw 18 is known to be embedded in a large neutral HI cloud extending over several kpc (van Zee et al. 1998). Furthermore, the strength of the Ly $\alpha$ absorption clearly decreases from the center to the border of the NW, as shown in Fig. 2 for slits 4 to 7 (slits 3 to 1 show also a slight decrease), corresponding to an apparent change of $N_{\mathrm{HI}}$ by up to a factor of $\sim 10$. This systematic change of the $\operatorname{Ly} \alpha$ will be explained below as the result of simple radiation transfer effects (Sect. 3.2.4).

Because of the configuration of their apertures, centered on the bright UV peak of the NW region, the GHRS and STIS MH03 spectra are in good agreement (cf. Fig. 3). This is also true for the central slit of the 2002 STIS data. The integrated spectrum of the NW region shows residual emission at the center that may stem from the contribution of the external slits that show such emission and to geocoronal $\operatorname{Ly} \alpha$ residuals, as the STIS MH03 and GHRS spectra have better resolution, which allows a more reliable correction. Since the profile shape in the wings remains the same, we take the STIS MH03 spectrum as a proxy for an integrated spectrum of the NW region in our modeling. We derived for this spectrum an HI column density of $\sim 2 \times 10^{21} \mathrm{~cm}^{-2}$ from Voigt profile fitting (cf. Table 2).

\subsection{Imaging}

The UV images, part of the same observing program GO9054 as that of the STIS spectroscopy, were retrieved from the ESO/ST-ECF archive. IZw 18 was observed with F25SRF2 filter with bandpass centered at $1457 \AA$. Standard calibrations were performed through CALSTIS pipeline. Images were then corrected for misalignment, divided by the exposure time, and co-added. The final FUV image was then multiplied by PHOTFLAM and PHOTBW header keywords to obtain a fluxcalibrated image.

We also retrieved HST optical images from the archive obtained with the Wide Field Planetary Camera 2 (WFPC2) under programs GO-6536 and GO-5434. Data consist of $\mathrm{H} \alpha$ and $\mathrm{H} \beta$ narrow-band imaging and corresponding broad band continuum images (see Table 1). Data were first processed through the standard HST pipeline that gives images in units of counts per second. Multiplying by PHOTFLAM keyword gives fluxes in erg s $\mathrm{s}^{-1} \mathrm{~cm}^{-2} \AA^{-1}$. Finally all images are rotated and aligned to the same orientation and co-added in each filter using inverse variance weighting. OWe estimated the line flux contribution to the continuum images using the appropriate filter throughput ratios at $\mathrm{H} \alpha$ and $\mathrm{H} \beta$ wavelengths and filter width given by PHOTBW. Continuum images were scaled and subtracted from online images, then multiplied by the filter bandwidth to obtain pure emission line fluxes. Continuum-subtracted $\mathrm{H} \alpha$ and $\mathrm{H} \beta$ images of IZw 18 are e.g. shown in Cannon et al. (2002). We measured a total $\mathrm{H} \alpha$ flux (uncorrected for reddening) of $3.28 \times$ $10^{-13} \mathrm{erg} \mathrm{s}^{-1} \mathrm{~cm}^{-2}$ within a circular aperture of $10.5^{\prime \prime}$ radius, in agreement with values found by de Mello et al. (1998) and Cannon et al. (2002).

Extinction: The extinction map of IZw 18 is created using the ratio between $\mathrm{H} \alpha$ and $\mathrm{H} \beta$ images. In the absence of dust extinction, the theoretical value of the Balmer ratio is known to be loosely sensitive to temperature and density. Following Cannon et al. (2002), we adopted a value of $\mathrm{H} \alpha / \mathrm{H} \beta=2.76$. Potential sources of error on the expected value, such as collisional excitation of HI or underlying stellar absorption, are also addressed in this paper. An $E(B-V)$ map was thus constructed using the relation:

$$
E(B-V)_{\mathrm{H} \alpha / \mathrm{H} \beta}=\frac{2.5 \times \log \left(2.76 / R_{\mathrm{obs}}\right)}{k\left(\lambda_{\alpha}\right)-k\left(\lambda_{\beta}\right)}
$$

where $R_{\mathrm{obs}}=f_{\mathrm{H} \alpha} / f_{\mathrm{H} \beta}$ is the absolute observed flux ratio, and $k\left(\lambda_{\alpha}\right), k\left(\lambda_{\beta}\right)$ are the extinction curve values at $\mathrm{H} \alpha$ and $\mathrm{H} \beta$ wavelengths. We adopt $k\left(\lambda_{\alpha}\right)-k\left(\lambda_{\beta}\right)=-1.08$, from Cardelli et al. (1989), which is appropriate for resolved galaxies and diffuse interstellar regions. Finally, the extinction value was corrected for galactic contribution following Schlegel et al. (1998), which accounts for $0.032 \mathrm{mag}$. The resulting extinction map is shown in Fig. 4.

Overall the extinction in IZw 18 and in its NW region is known to be very low. Mas-Hesse (1990) found that the Balmer decrement of the whole NW region is consistent with no extinction. However, Dufour et al. (1988) reported an extinction of $E(B-V) \sim 0.17$ in their $2.5^{\prime \prime} \times 6^{\prime \prime}$ slit. Ground-based spectroscopic observations revealed typical values ranging from $E(B-V) \sim 0.03$ up to 0.2 (e.g. Vílchez \& Iglesias-Páramo 1998; Izotov et al. 1997; Martin 1996). The main reasons for such discrepancies may be differences in the aperture size and the location of the slits on the galaxy, as the dust does not seem to be homogeneously distributed in IZw 18 (see Fig. 4). In the present work, we define a circular aperture (3.2" radius) centered on the NW region. We exclude the central region $\left(1^{\prime \prime}\right.$ circular aperture), where $\mathrm{H} \alpha$ and $\mathrm{H} \beta$ emissions are much weaker and the Balmer ratio gives unreasonably low values, from our measurement. The mean color excess derived in this way is $E(B-V) \approx 0.042$. This value agrees with the determinations by Cannon et al. (2002) $(E(B-V)=0-0.09)$, obtained in different parts of the NW region, and with Péquignot (2008). We also find no extinction when the central region is not excluded, in agreement with Mas-Hesse (1990). Subsequently we will adopt an average value of $E(B-V)=0.05$ for the NW region.

Intrinsic Ly $\alpha$ emission: From the $\mathrm{H} \alpha$ image and with the extinction map, we produced a theoretical Ly $\alpha$ emission map (Fig. 4). It is determined using $f(\operatorname{Ly} \alpha)=8.7 \times f(\mathrm{H} \alpha) \times$ $10^{(1.048 \times E(B-V))}$, where we assume a case $\mathrm{B}$ recombination theory (Brocklehurst 1971) and the extinction law previously mentioned. Naturally, given the small extinction corrections, the resulting map of intrinsic $\operatorname{Ly} \alpha$ emission shows a very similar morphology to the $\mathrm{H} \alpha$ map. Schematically, the NW region is surrounded by a Ly $\alpha$ emission shell including in particular one bright knot (NW1 in the notation of Cannon et al. 2002).

Finally, the spatial variation of the UV continuum, and the intrinsic Ly $\alpha$ equivalent width are shown in Fig. 5. The UV image has been corrected for reddening, scaled to Ly $\alpha$ wavelength using a UV slope of $\beta \sim-2$, and used with the intrinsic Ly $\alpha$ map 

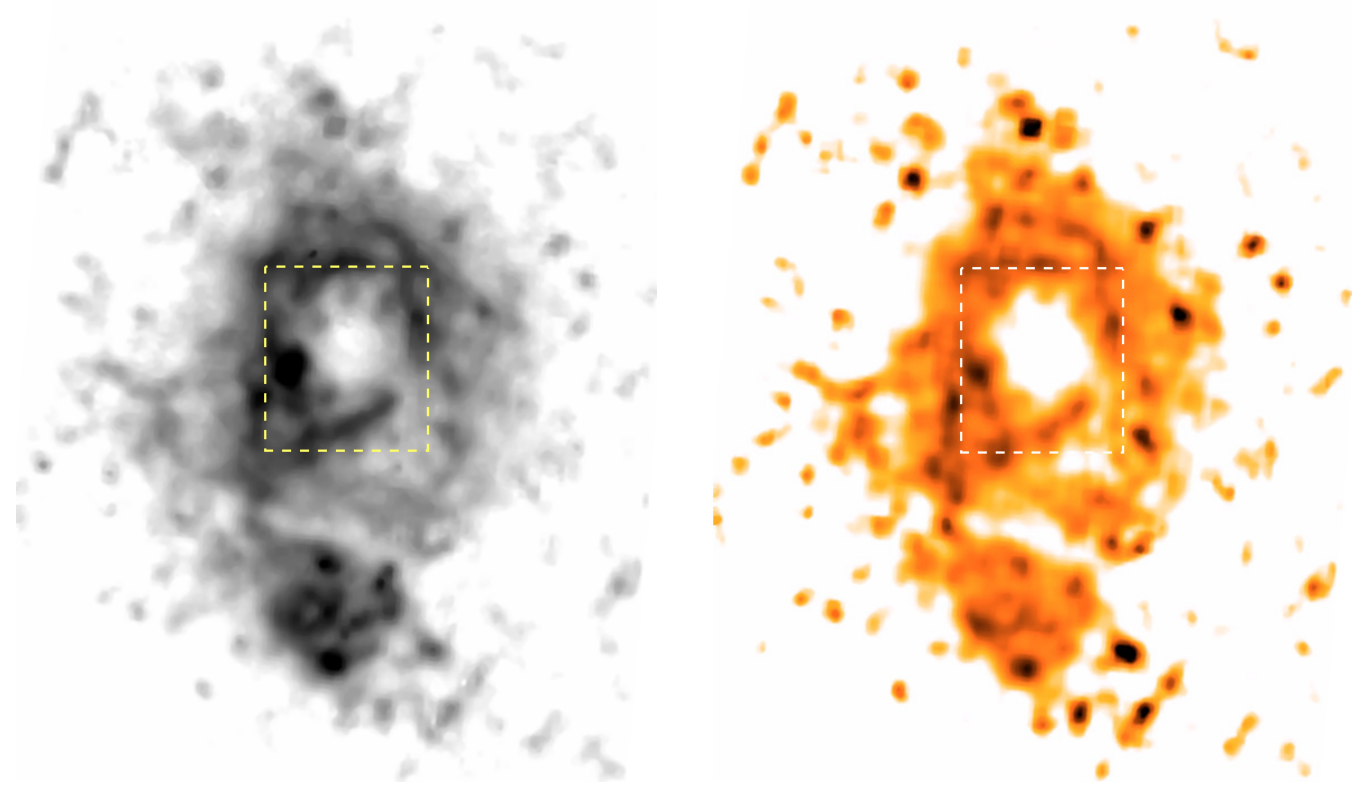

Fig. 4. IZw 18 imaging. Left: intrinsic Ly $\alpha$ emission map on a logarithmic scale, obtained by correcting the observed H $\alpha$ image with the extinction map and assuming case B recombination theory (see text for details). The result has been smoothed using a median filter (width $=5$ ). Right: shows the extinction map, on a linear scale, obtained from the Balmer decrement $\mathrm{H} \alpha / \mathrm{H} \beta$ then median-filtered (width $=5$ ). Inverted color scale is used showing higher emission and dust content in darker color. The NW integration box (cf. Fig. 1) is also shown by a dashed line. The size of the field of view is about $13^{\prime \prime} \times 15^{\prime \prime}$ and the orientation is the same as in Fig. 1 .

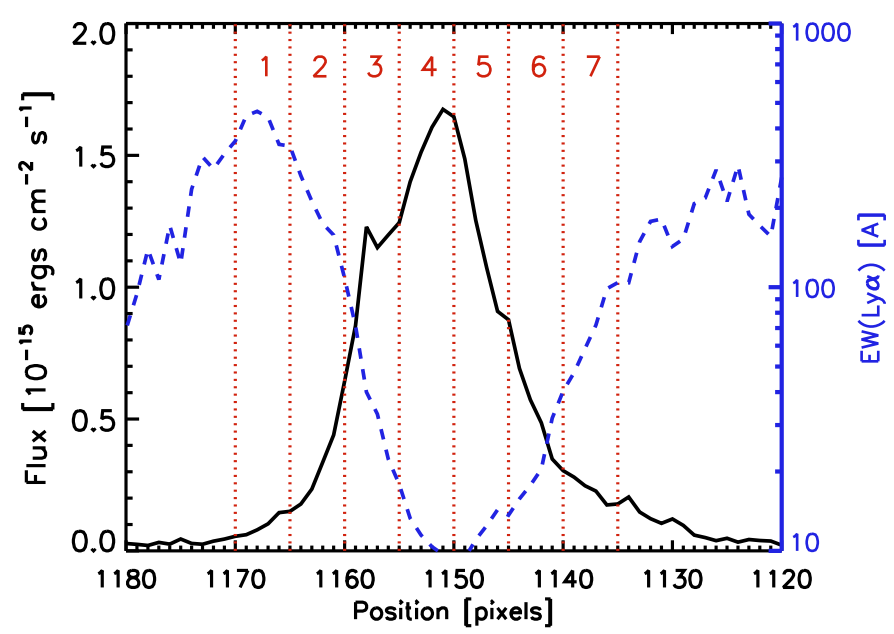

Fig. 5. 1D emission profile of NW region. The different profiles are obtained by adding the flux along the slit (spatial direction) in the same aperture as used for the extraction of the spectra (cf. Fig. 2) centered on the NW region. Then the 1D profile corresponds to the direction along the dispersion axis. One pixel corresponds to $\sim 0.1^{\prime \prime}$. The dark solid curve is the $1500 \AA$ flux represented on the left $y$-axis, and blue dashed line the intrinsic Ly $\alpha$ equivalent width represented on logarithmic scale on the right axis. The position of the seven adjacent slits are also marked, showing the spatial variation in the emission between the different slits.

to construct the theoretical $E W_{\mathrm{Ly} \alpha}$ map. Over the entire NW region we obtain $E W_{\mathrm{Ly} \alpha} \sim 50 \AA$, compatible with expectations for a young starburst. However, as shown in Fig. 5, we observe very high values around the UV-bright central region.

Taken together, the observations of strong Ly $\alpha$ absorption across the entire NW region despite the presence of intrinsic strong Ly $\alpha$ emission and a very low amount of extinction clearly call for a physically consistent explanation of these apparent contradicting phenomena.

\subsection{Other observational constraints}

A mean velocity offset, $\Delta v(\mathrm{em}-\mathrm{abs})$, between the systemic velocity, measured from the optical lines, and metallic absorption lines of O I and Si II, was measured by Kunth et al. (1998) in the small GHRS aperture centered on the NW region. They found $\Delta v(\mathrm{em}-\mathrm{abs}) \sim 25 \mathrm{~km} \mathrm{~s}^{-1}$, indicating that the neutral gas is mostly static with respect to the central HII region. Recent FUSE observations including other ISM absorption lines confirm the absence of an outflow in IZw 18 on a large aperture including by far all the UV emitting regions of this galaxy; Grimes et al. (2009) measure velocity shifts between $\sim 0$ and $40 \mathrm{~km} \mathrm{~s}^{-1}$ with a mean offset of $8 \mathrm{~km} \mathrm{~s}^{-1}$.

The Doppler parameter $b$ describes the thermal motion of hydrogen atoms. The HI velocity dispersion observed by van Zee et al. (1998) is about $12-14 \mathrm{~km} \mathrm{~s}^{-1}$, which translates to $b \simeq 17-$ $20 \mathrm{~km} \mathrm{~s}^{-1}$. A slightly higher value $\left(b \simeq 27 \mathrm{~km} \mathrm{~s}^{-1}\right)$ was quoted by Kunth et al. (1994) from their VLA observations. Given the very damped profile of the Ly $\alpha$ absorption, variations within this range of values does not affect the model fit.

The full width at half maximum $(F W H M)$ of the Ly $\alpha$ emission line can be constrained using $F W H M(\mathrm{H} \alpha)$. Dufour et al. (1988) found $F W H M(\mathrm{H} \alpha) \sim 6.1 \AA\left(280 \mathrm{~km} \mathrm{~s}^{-1}\right)$ from their spectrophotometry observations but with a resolution of $275 \mathrm{~km} \mathrm{~s}^{-1}$. Observations with a better resolution $\left(R \sim 11 \mathrm{~km} \mathrm{~s}^{-1} F W H M\right)$ indicates $F W H M(\mathrm{H} \alpha) \sim 150 \mathrm{~km} \mathrm{~s}^{-1}$ (Martin 1996). This is consistent with a relatively narrow emission line, and we adopt $F W H M(\operatorname{Ly} \alpha)=100 \mathrm{~km} \mathrm{~s}^{-1}$, although our results are insensitive to the differences found in the observations.

\section{Explaining the Ly $\alpha$ absorption in IZw 18}

\subsection{General considerations}

To transform the intrinsic Ly $\alpha$ emission (emitted in the HII region) to a pure absorption profile can in principle only be achieved in two ways: 1) by true destruction of Ly $\alpha$ photons (by 


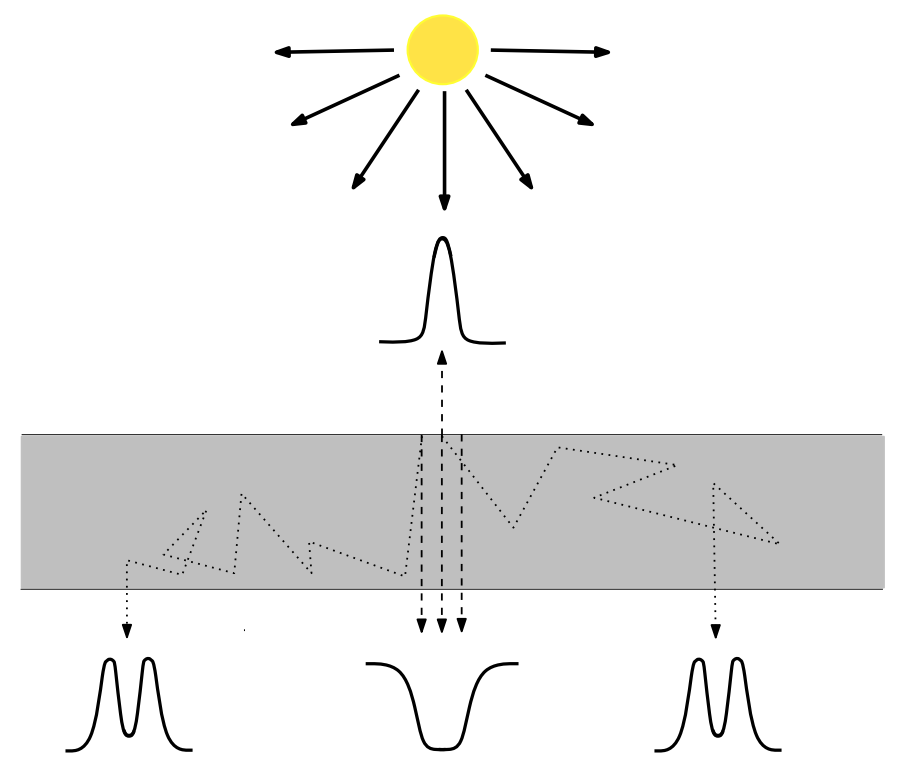

Fig. 6. Sketch showing geometrical effects on the Ly $\alpha$ profile shape for a point source behind a homogeneous dust-free slab emitting pure UV continuum radiation (flat continuum around $\operatorname{Ly} \alpha$ ). The output spectra represent the observed profiles in different regions and in the observer's line of sight perpendicular to the slab. The reflected spectrum by the slab is also represented.

dust or possibly by conversion to two-photon continuous emission in the ionized region), or 2) by geometrical effects leading to the scattering of $\operatorname{Ly} \alpha$ photons out of the line of sight, or by a combination of both.

Examples of line profiles due to dust absorption are shown e.g. in Verhamme et al. $(2006,2008)$ and Schaerer \& Verhamme (2008). Effect 2) is illustrated in Fig. 6, showing how for example even a dust-free slab produces an absorption (Voigt) profile along the central line of sight from a point-like background source. This geometrical situation also corresponds to the "classical" case of damped Ly $\alpha$ systems (DLA) in front of distant quasars or to other Ly $\alpha$ forest observations. If the scattering foreground layer was truly dust-free, it is clear that the photons are conserved; hence, the photons scattered away from line center (causing the apparent absorption line) must emerge somewhere. In a static configuration, radiation transfer effects redistribute the photons into the wings, leading to a symmetric double peak Ly $\alpha$ profile (Neufeld 1990), as sketched in Fig. 6 for the distant, noncentral lines of sight. Adding dust to effect 2), i.e. combining 1) and 2), will reduce the strength of the scattered component and further increase the depth of the central absorption profile.

Using radiation transfer models we now examine whether these effects can quantitatively explain the observations of IZw 18 and which of these effects is dominant.

\subsection{Ly $\alpha$ and UV continuum radiation transfer modeling}

\subsubsection{MCLya code and input parameters}

We use an improved version of the Monte Carlo radiation transfer code MCLya of Verhamme et al. (2006) including the detailed physics of Ly $\alpha$ line and UV continuum transfer, dust scattering, and dust absorption for arbitrary 3D geometries and velocity fields. The following improvements have been included (see Hayes et al. 2009, for more details): angular redistribution functions taking quantum mechanical results for $\operatorname{Ly} \alpha$ into account (cf. Dijkstra \& Loeb 2008; Stenflo 1980), frequency changes of Ly $\alpha$ photons due to the recoil effect (e.g. Zheng \& Miralda-Escudé 2002), the presence of deuterium (assuming a canonical abundance of $D / H=3 \times 10^{-5}$, Dijkstra et al. 2006), and anisotropic dust scattering using the Henyey-Greenstein phase function (using parameters adopted in Witt \& Gordon 2000). Furthermore, a relatively minor bug in the angular redistribution of Ly $\alpha$ photons has been fixed, and the code has been parallelized for efficient use on supercomputers. For the physical conditions in the simulations used for the present paper, these improvements lead only to minor changes with respect to the MCLya version used by Schaerer \& Verhamme (2008) and Verhamme et al. (2008). More details on the code upgrade will be given in Hayes et al. (2009).

For simplicity, and given the available observational constraints, all simulations carried out subsequently assume a homogeneous and co-spatial distribution of neutral hydrogen and dust with a constant density and temperature. The corresponding microscopic HI velocity distribution is described by the Doppler parameter $b$. The remaining input parameters of the code are the HI geometry and velocity field. We also consider the spatial location and distribution of both the UV continuum and line emission source(s).

We consider the following HI geometries: spherically symmetric shells with a central source, and plane-parallel slabs with a background or internal source (including different source geometries). These cases are described by 3 additional parameters: $(i)$ the expansion velocity of the shell, $v_{\exp }$, or the velocity of the slab with respect to the source; (ii) the HI column density towards the source, $N_{\mathrm{HI}}$; and (iii) the dust-absorption optical depth $\tau_{\mathrm{a}}$ that expresses the dust-to-gas ratio. As discussed by Verhamme et al. (2006), $\tau_{\mathrm{a}}$ is related to the usual color excess $E(B-V)$ by $E(B-V) \approx(0.06 \ldots . .0 .11) \tau_{\mathrm{a}}$; we assume $E(B-V)=0.1 \tau_{\mathrm{a}}$ for convenience. In short, for a given geometry we have 4 parameters $\left(b, v_{\text {exp }}, N_{\mathrm{HI}}, \tau_{\mathrm{a}}\right) ; b=20 \mathrm{~km} \mathrm{~s}^{-1}$ and $v_{\exp } \approx 0 \mathrm{~km} \mathrm{~s}^{-1}$ are constrained by the observations (see Sect. 2.3), $\tau_{\mathrm{a}}$ is varied between 0 (no dust) and 0.5 , the maximum allowed by the observations (Sect. 2.3), and $N_{\mathrm{HI}}$ is varied to reproduce the observed Ly $\alpha$ line profile.

For each parameter set, a full Monte Carlo simulation is run allowing for enough statistics to compute both integrated and spatially resolved spectra in the Ly $\alpha$ region. The radiation transfer calculations cover a broad enough spectral range (here typically from -10000 to $+10000 \mathrm{~km} \mathrm{~s}^{-1}$ ) to reach the continuum for the highest column-density simulations. As described in Verhamme et al. (2006) our MC simulations are computed for a flat input spectrum, keeping track of the necessary information to recompute a posteriori simulations for arbitrary input spectra. For the Ly $\alpha$ fits we assume an input spectrum given by a flat (stellar) continuum plus the Ly $\alpha$ line, described by a Gaussian with variable equivalent width $E W_{\mathrm{Ly} \alpha}$ and full width at half maximum $F W H M(\operatorname{Ly} \alpha)$. The $E W_{\mathrm{Ly} \alpha}$ is kept free, although constraints are available from our theoretical (intrinsic) Ly $\alpha$ map; an $F W H M=100 \mathrm{~km} \mathrm{~s}^{-1}$ is assumed as for $\mathrm{H} \alpha$, although our results are basically independent of its exact value. Other continua, such as synthetic high-resolution starburst spectra from Schaerer \& Verhamme (2008), can also be used.

\subsubsection{Shell models}

To consider a simple geometry to understand the observed Ly $\alpha$ absorption of IZw 18, we examine predictions for the integrated spectrum of a spherical shell with/without dust. In this case no "loss" of photons by spatial diffusion is allowed; hence, to 


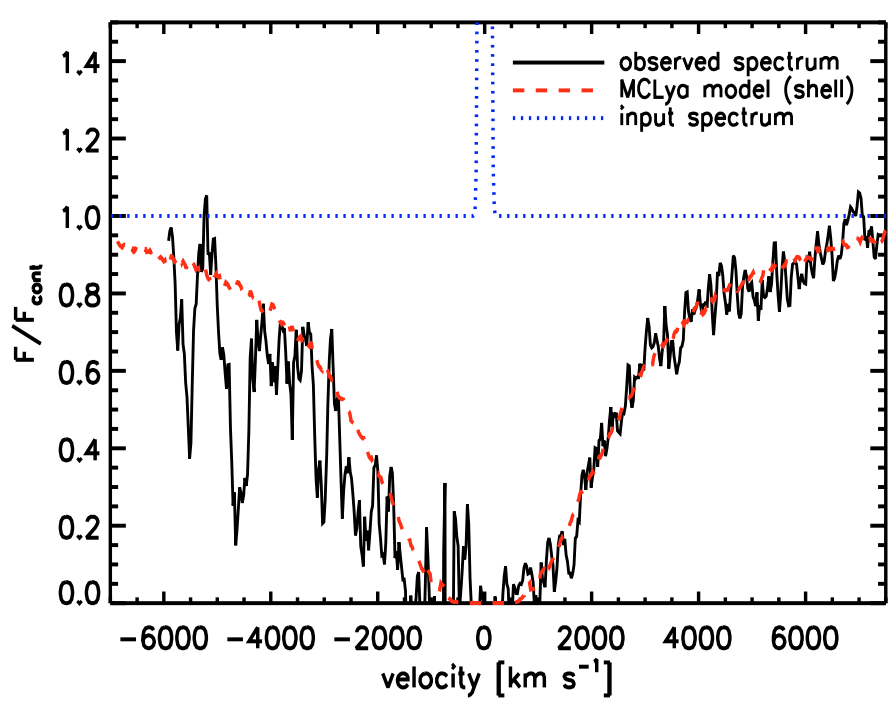

Fig. 7. Comparison of the observed and fitted $\operatorname{Ly} \alpha$ profile of IZw 18 assuming a spherical shell model. The observed STIS spectrum (from MH03) is represented by the dark line. The model fit, using a shell geometry, is plotted with red dashed line and the parameters used are $N_{\mathrm{HI}}=6.5 \times 10^{21} \mathrm{~cm}^{-2}, \tau=0.5, v=0 \mathrm{~km} \mathrm{~s}^{-1}, b=20 \mathrm{~km} \mathrm{~s}^{-1}$. The blue dotted line represents the input spectrum of the simulation. It consists of a flat UV continuum plus a Gaussian Ly $\alpha$ emission line with $F W H M=100 \mathrm{~km} \mathrm{~s}^{-1}$. The intrinsic equivalent width adopted is $E W(\operatorname{Ly} \alpha)=60 \AA$.

transform intrinsic Ly $\alpha$ emission into an absorption profile requires absorption by dust. We now examine whether spatially integrated shells can recover the observed profile for reasonable amounts of dust and reasonable HI columns.

Adopting an average extinction of $E(B-V) \approx 0.05$ (i.e. $\tau_{\mathrm{a}}=$ $0.5)$ and $b=20 \mathrm{~km} \mathrm{~s}^{-1}$ we computed several static shell models with varying $N_{\mathrm{HI}}$. As shown in Fig. 7 , the predicted profile agrees with the observations for $N_{\mathrm{HI}}=6.5 \times 10^{21} \mathrm{~cm}^{-2}$ and for an input spectrum with a Ly $\alpha$ line equivalent width $E W(\operatorname{Ly} \alpha)=60 \AA$. We do not fit the absorptions in the blue wing, attributed to Si II $\lambda \lambda$ 1193.3, 1194, 5 and Si III $\lambda \lambda$ 1206.5, 1207.5 (Schaerer \& Verhamme 2008) and possibly to Galactic and intergalactic HI absorption, since these are not taken into account in our model.

The reason for the resulting broad damped Ly $\alpha$ absorption is as follows: because of the high HI column density, even a small amount of dust destroys almost all photons in and around the Ly $\alpha$ line center. Scattering on hydrogen atoms with such a high column density greatly increases the mean path of Ly $\alpha$ photons, hence the probability of being absorbed by dust. Therefore, the net absorption is only caused by dust absorption, since in the present case, we observe all the scattered photons escaping from the shell, without any line-of-sight effect.

Influence of $\boldsymbol{V}_{\text {exp }}$ : we adopted a static shell in our model to fit the Ly $\alpha$ absorption profile. As discussed in Verhamme et al. (2006), for increasing $v_{\exp }$ more $\operatorname{Ly} \alpha$ photons will escape from the red part of the line, because Ly $\alpha$ photons are already seen redshifted by hydrogen atoms. However, since the high column density reduces the escape probability, we can vary the expansion velocity in a certain range without affecting the quality of the fit. The highest velocity allowed is around $50 \mathrm{~km} \mathrm{~s}^{-1}$, which already exceeds the observed outflow velocity of IZw 18 .

Influence of $\boldsymbol{E} \boldsymbol{W}_{\mathrm{Ly} \alpha}$ : we can show that $E W_{\mathrm{Ly} \alpha}$ close to the maximum value expected by synthesis models (Schaerer 2003), for normal IMF populations, are allowed in our model to fit IZw 18 profile. Indeed, when we use an input Ly $\alpha$ line with $E W_{\mathrm{Ly} \alpha}=$ $200 \AA$ the fit remains globally unchanged. Again, in spite the large damped absorption, high intrinsic $E W_{\mathrm{Ly} \alpha}$ is not excluded by radiation transfer simulations because of the high HI column density.

Other solutions: we need to invoke a relatively high column density $\left(N_{\mathrm{HI}}=6.5 \times 10^{21} \mathrm{~cm}^{-2}\right)$ to obtain a good fit of the absorption wings. On the other hand, all photons that, in reality, will scatter away from the observer's line of sight are recovered in our simulation, since we integrate over the entire surface of the shell. Relaxing this assumption, i.e. considering different geometries, would in particular also allow us to lower $N_{\mathrm{HI}}$.

The solution proposed here to fit IZw 18 profile is not unique, and different combinations of $N_{\mathrm{HI}}$ and $\tau_{\mathrm{a}}$ can reproduce the absorption. For instance, the use of a higher value for the extinction $\left(\tau_{\mathrm{a}}=1\right)$ and a lower HI column density $\left(N_{\mathrm{HI}}=5 \times 10^{21} \mathrm{~cm}^{-2}\right)$ produce the same fit quality. Overall, this somewhat academic case of a shell model for IZw 18 serves to show that even low dust quantities may suffice to transform Ly $\alpha$ emission into a broad absorption profile, provided a sufficiently high column density and/or sufficiently low outflow velocity, as also discussed in Sect. 4. In any case, the radio observations of IZw 18 very clearly show a large spatial extension of HI compared to the size of the NW region (and to that of the spectroscopic apertures). The effect of such geometries on Ly $\alpha$ are addressed now.

\subsubsection{Extended geometries and line of sight effects}

The galaxy spectrum we observe in reality, could deviate significantly from the simple homogeneous shell model presented here, since the source is spatially resolved and the spectrum is not integrated over the whole shell surface. Furthermore, the spectrum can depend on viewing angle and on the geometry of the ISM.

We show in Fig. 8 that IZw 18 absorption can be adjusted with lower Hi column density than required for the shell model and without any dust $(E(B-V)=0)$. This result is achieved by taking a slab geometry with a static gas and applying sightline selection criterium, where only photons in the observer's direction are collected. Then the absorption is caused not by dust destruction, but by diffusion of the photons out of the observer's direction. Strictly speaking, no photon is destroyed. This demonstrates even better that Ly $\alpha$ absorption can be observed in dustfree galaxies (cf. Fig. 6 for a schematic overview). Only a nearly static neutral ISM is required, with $N_{\mathrm{HI}} \sim 3 \times 10^{21} \mathrm{~cm}^{-2}$ in this case.

We expect to recover the diffused photons in other directions and/or farther from the source. On the other hand, in the presence of dust, this diffuse part would be attenuated or suppressed. For example, for models with homogeneous gas and dust distributions, our Ly $\alpha$ transfer simulations (see Hayes et al. 2009) already predict quite low escape fractions for Ly $\alpha$ line photons, with $f_{\text {esc }}$ typically $5-10 \%$ for column densities $N_{\mathrm{HI}} \gtrsim 10^{21} \mathrm{~cm}^{-2}$, dust optical depths $\tau_{\mathrm{a}}=0.2$, and low expansion velocities $\left(v_{\text {exp }} \lesssim 50 \mathrm{~km} \mathrm{~s}^{-1}\right)$. Much lower escape fractions $\left(f_{\text {esc }} \sim 10^{-3 \ldots-4}\right)$ are predicted for larger amounts of dust, such as for the average value adopted for the NW region; therefore, we expect relatively small amounts of diffuse emission from Ly $\alpha$ line photons. 


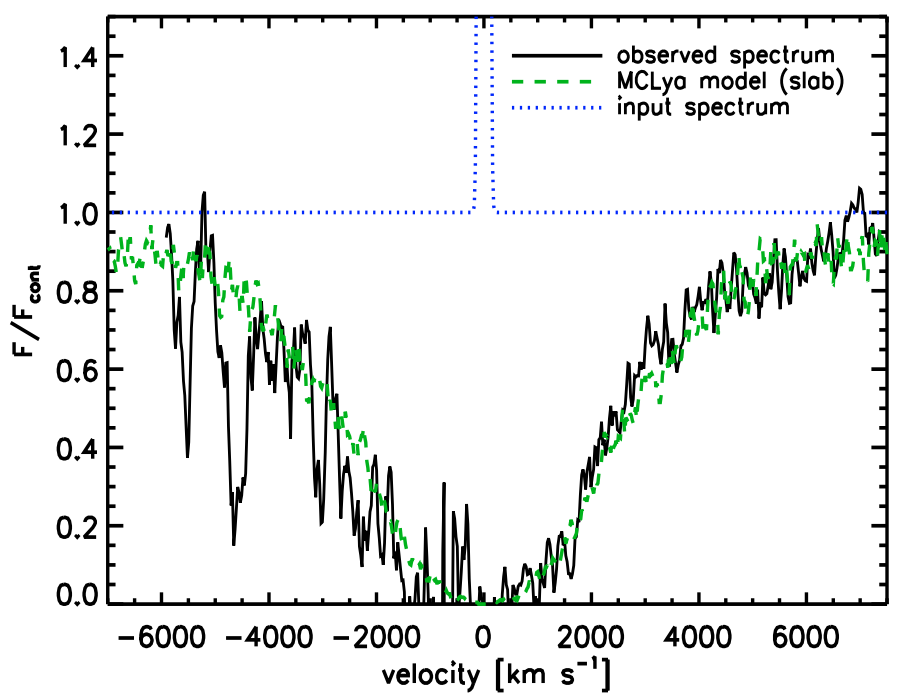

Fig. 8. Ly $\alpha$ absorption fitting II. The observed spectrum (STIS MH03) is represented by the dark line. A slab geometry is used for the model spectrum and only photons in the observer's sightline are collected. It is plotted with green dashed line and the parameters used are $N_{\mathrm{HI}}=$ $3 \times 10^{21} \mathrm{~cm}^{-2}, \tau=0$ (no dust), $v=0 \mathrm{~km} \mathrm{~s}^{-1}, b=20 \mathrm{~km} \mathrm{~s}^{-1}$. The blue dotted line represents the input spectrum of the simulation. It consists of a flat UV continuum plus a Gaussian Ly $\alpha$ emission line with $F W H M=$ $100 \mathrm{~km} \mathrm{~s}^{-1}$. The intrinsic equivalent width adopted is $E W(\operatorname{Ly} \alpha)=60 \AA$.

\subsubsection{Spatial variations of $\operatorname{Ly} \alpha$ profile}

In Sect. 2.1 (Fig. 2) we have shown that the Ly $\alpha$ profile shows spatial variations between the different STIS slits. We now demonstrate that, given the observational constraints, the Ly $\alpha$ radiation transport explain these variations fairly well. We consider a large, static, and uniform cloud of $\mathrm{HI}\left(N_{\mathrm{HI}}=3 \times\right.$ $\left.10^{21} \mathrm{~cm}^{-2}, b=20 \mathrm{~km} \mathrm{~s}^{-1}, \tau_{\mathrm{a}}=0.5\right)$ represented by a slab geometry, covering the NW star-forming region. We then simulate the observed spatial variations of the emission strength by using weighted point sources located in front of the HI slab, emitting a flat UV continuum, as input to our radiation transfer code following the observed UV profile of Fig. 5. The addition of Ly $\alpha$ line emission will be discussed below.

The result of this simulation is shown in Fig. 9. The output spectrum is what an observer would see when his line-of-sight is perpendicular to the slab surface. At the center $(1150 \leq$ pixel $\leq$ 1155 ), in the direction of the brightest source, we observe the strongest (largest) Ly $\alpha$ absorption profile. The profile proves increasingly narrower as one moves away from the center, what reproduces the trend observed in IZw 18. The double-peak contribution, characteristic of diffused photons, can even be seen in the peripheral region $(1135 \leq$ pixel $\leq 1140)$.

To understand these results, let us decipher the different contributions in the simulation. Figure 6 depicts the situation for this purpose. It shows the observed spectra in a simulation using a point source and isotropic emission behind a uniform slab of neutral gas. Observing the slab face-on, toward the source, we obtain an absorption profile. Only photons far from the line center are transmitted directly, forming the "continuum". Photons in the line center are resonantly absorbed and reemitted, diffusing in frequency and in space, and leading to the lack of emission at and around the line core. These photons will be collected if we look at the cube at a position far from the source. A double-peak profile is then observed consisting of the diffused photons and

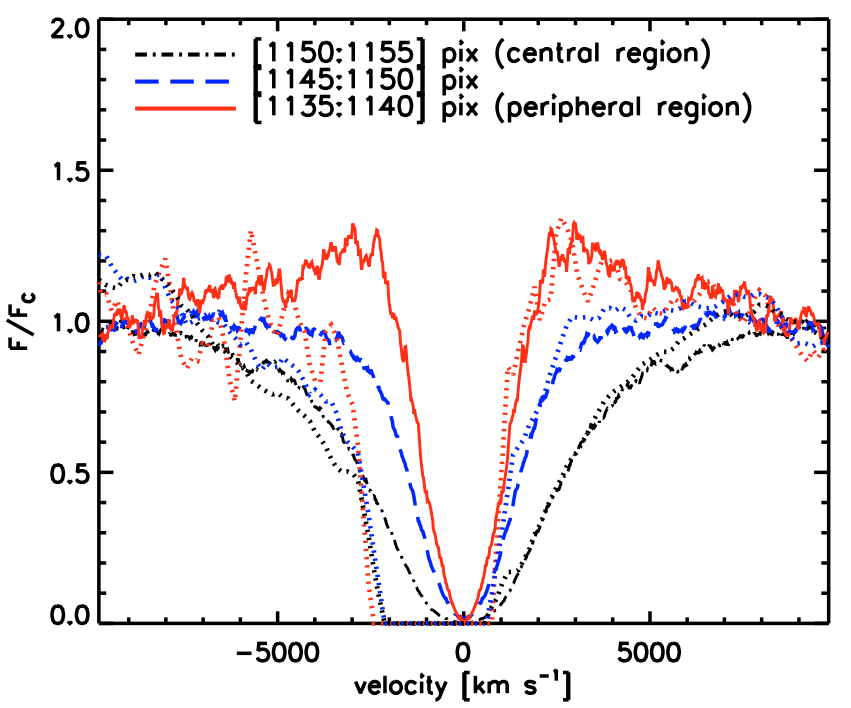

Fig. 9. Predicted spatial variations of the $\operatorname{Ly} \alpha$ profile. The simulation consists of an homogeneous HI slab with $N_{\mathrm{HI}}=3 \times 10^{21} \mathrm{~cm}^{-2}$ and $\tau_{\mathrm{a}}=$ 0.5 illuminated with an isotropic, extended, UV source emitting a flat continuum. The emission strength spatially varies from the center to the edge to reproduce the observed UV surface brightness of IZw 18. An observer line-of-sight perpendicular to the cube is chosen $(\theta=0)$. The different plotted profiles then correspond to different regions at the surface of the slab and are marked in units of pixels corresponding to the position of the slits in Fig. 5. The observed profiles (cf. Fig. 2) are plotted with dotted lines. The blue wing of the profiles is not well-fitted because it is affected by geocoronal emission and HI Galactic absorption, which the model does not account for.

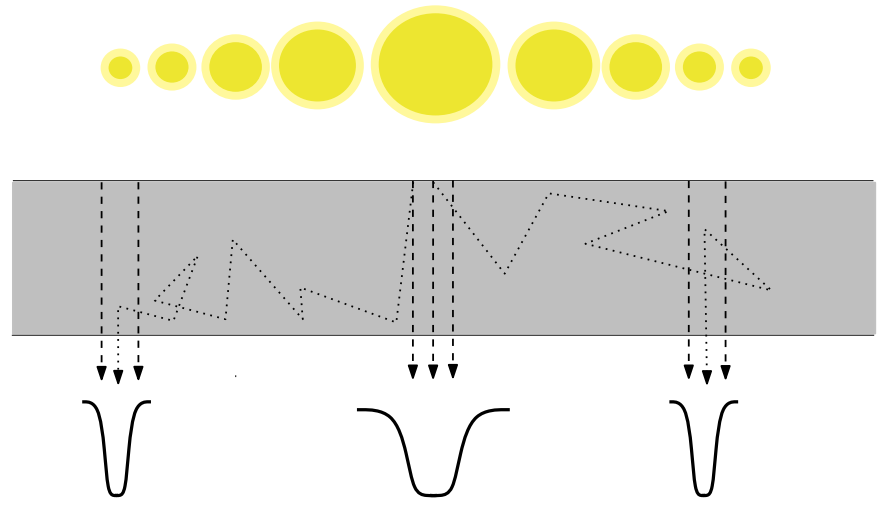

Fig. 10. Geometrical effects on the $\operatorname{Ly} \alpha$ profile shape: homogeneous, static, and dust-free slab of neutral gas illuminated by a series of isotropic point sources emitting UV continuum radiation centered on Ly $\alpha$ wavelength. An extended source is simulated in this case with a varying emission strength, symbolized by the size of the individual point sources. The output spectra represent the observed profiles in different regions and in the observer's line of sight perpendicular to the slab.

the absence of photons that would have escape directly, without scattering, in this direction.

Figure 10 now shows a combination of these single sources but with different intensities, illustrating the extended source simulation of Fig. 9. As for the single source, the spectrum of the central region shows a typical damped absorption. At the positions of the fainter peripheral sources, two contributions lead to a narrower absorption profile: a) the transmitted flux that is fainter than in the central region; and b) photons that have diffused from the brighter sources to escape farther (double-peak emission), contributing to "filling the wing". In the central region 
the direct transmission is stronger and the diffuse part is weaker. In this way spatial variations of the UV continuum combined with the resonant transport effects of Ly $\alpha$ radiation, can explain qualitatively the observed Ly $\alpha$ profile variations in IZw 18 . The observed profile in slit 7 (Fig. 2) may even show a hint of the predicted double-peak profile in its red wing, although the $\mathrm{S} / \mathrm{N}$ is quite low in this region.

For the effect of adding $\operatorname{Ly} \alpha$ line emission on top of the UV continuum emission, one could naively expect a very different behavior given the very large $\operatorname{Ly} \alpha$ equivalent width of the source in the peripheral parts of the NW region (cf. Fig. 5). However, the final spectrum remains unchanged despite the high Ly $\alpha$ equivalent width used. It appears that the photons emitted at the core of the line are either destroyed by dust ( $\tau_{\mathrm{a}}=0.5$ here) or backscattered, and only photons with higher frequency shift diffuse and contribute to the double-peak emission. Therefore, increasing $E W_{\mathrm{Ly} \alpha}$ has no incidence on the output spectrum since with $F W H M(\operatorname{Ly} \alpha)=100 \mathrm{~km} \mathrm{~s}^{-1}$, all photons are emitted close to the center. This is easily confirmed by looking at the reflected spectrum (cf. Fig. 6) which increases with higher $E W_{\mathrm{Ly} \alpha}$. We need to use unreasonably high $F W H M(\operatorname{Ly} \alpha)\left(\gtrsim 1000 \mathrm{~km} \mathrm{~s}^{-1}\right)$ to affect our result and see the double-peak contribution increasing (in the profile wings). This implies in particular that our model predictions are insensitive to the observed spatial variations of $E W_{\text {Ly } \alpha}$ (cf. Fig. 5).

In short, we conclude that the observed variations in the Ly $\alpha$ profile across the NW region can be understood by a combination of the line-of-sight effects discussed earlier and by radiation transfer effects related to an extended source.

\subsection{Discussion}

For the first attempt to reproduce the damped absorption profile of IZw 18, we used a simple expanding-shell model (Sect. 3.2.2). If line-of-sight arguments could not be invoked, we would need a relatively high column density $\left(N_{\mathrm{HI}}=6.5 \times 10^{21} \mathrm{~cm}^{-2}\right)$ and a minimum amount of dust $(E(B-V) \sim 0.05)$, which in this case is the only way to lose Ly $\alpha$ photons. However, when we spatially selected photons in the observer's sightline, we showed (Fig. 8) that one may observe Ly $\alpha$ in absorption even without any dust $(E(B-V)=0)$. These conclusions also held for the SE region of IZw 18 for which the integrated spectrum show slightly more Ly $\alpha$ absorption (a Voigt fit yields $N_{\mathrm{HI}} \sim 4 \times 10^{21} \mathrm{~cm}^{-2}$ ).

Martin (1996) finds evidence of supergiant shell in IZw 18 expanding at a speed of $35-60 \mathrm{~km} \mathrm{~s}^{-1}$. The geometry proposed is a bipolar shell seen almost perpendicularly to its main axis (cf. their Fig. 4). This configuration is comparable to the shell geometry adopted here (Sect. 3.2.2) given the negligible effects of such small expansion velocities on our model spectrum. However, the output spectrum of the shell model would be significantly affected if the HI coverage is inhomogeneous and low column densities are observed in some sightlines, which is still unclear here. For the same reasons (low velocity and large HI coverage), applying our extended geometry scenario (Sect. 3.2.3) to this configuration would yield the same results, because our sightline selection is still compatible with this ISM morphology.

From our shell model we derived an HI column density of $6.5 \times 10^{21} \mathrm{~cm}^{-2}$ that is higher than any independent measurements of $N_{\mathrm{HI}} \sim 2.6 \times 10^{21} \mathrm{~cm}^{-2}$ from radio data by van Zee et al. (1998). Since radio observations measure the total HI content, our model would imply, for symetrical reasons, a value twice higher than given. However, with a typical beam size of $5^{\prime \prime}$, the radio observations are not able to resolve the potential subparsec-size HI clumps in the NW region, and the smoothing effects could lead easily to an underestimation by a factor of 2-4. Therefore, in the absence of higher resolution observations, we cannot rule out the supershell geometry.

The second model adopted led to a good fit with $N_{\mathrm{HI}}=$ $3 \times 10^{21}$ and without dust comparable to observational constraints, and also explained the spatial variations of the absorption profile. It is therefore more likely that, if the emission region is embedded in an HI region, the geometry would be not symmetric, with a higher column density in the front and/or ionized holes in the back. Finally, it is worth noting that the geometry proposed by Martin (1996) is not an embedded-like source, and the expanding shell is bipolar and asymmetric with an axis inclined by $i \sim 10^{\circ}$ to our line of sight (see their Fig. 4), while van Zee et al. (1998) find a higher inclination of $i \sim 55^{\circ}$.

\section{Comparison of IZw 18 with other nearby and high-z starbursts}

We have just shown how with a low extinction or even no dust at all it is possible to explain by radiation transfer and geometrical effects the transformation of a strong intrinsic Ly $\alpha$ emission into the broad Ly $\alpha$ absorption profile observed in IZw 18 . We need now to understand whether this galaxy is unique or representative of a certain class of objects and what our results imply for other studies, including $\operatorname{Ly} \alpha$ observations of high- $z$ objects in particular.

\subsection{Comparison with local starbursts}

Four of the eight HII galaxies observed with GHRS/HST by Kunth et al. (1998) show broad Ly $\alpha$ absorption profiles: II Zw 70, Mrk 36, SBS 0335-052, and IZw 18. As already noted by these authors, these objects clearly differ from those with Ly $\alpha$ in emission by very low velocity shifts between the interstellar absorption lines and the systemic velocity ${ }^{2}$. An essentially static ISM appears therefore as one of the main factors leading to Ly $\alpha$ absorption, as already concluded by these authors and as supported by our radiation transfer modeling.

Furthermore, among the Ly $\alpha$ absorbers, SBS 0335-052 and II Zw 70 clearly show higher extinction, with $E(B-V)=0.18$ and 0.15 , respectively (less than 0.02 for Mrk 36, Izotov \& Thuan 1998). Hence the ISM properties of these objects should fulfill the same conditions, which have allowed us to explain the Ly $\alpha$ absorption of IZw 18, and the dust destruction of Ly $\alpha$ photons should be equally or more important. Although very likely, we cannot fully prove this until HI column density measurements from the radio are available for all of them. For SBS 0335$052 N_{\mathrm{HI}}$ reaches high values up to $\$ 9.4 \times 10^{20} \mathrm{~cm}^{-2}$ (Pustilnik et al. 2001). Similarly, Mrk 36 shows a high column-density peak up to $2.4 \times 10^{21} \mathrm{~cm}^{-2}$ (Bravo-Alfaro et al. 2004). Of course, depending on the efficiency of dust destruction, some spatial regions with diffuse Ly $\alpha$ emission may be expected; however, this is not necessarily the case. For example, for SBS 0335-052 we know that Ly $\alpha$ absorption is observed over a large area, showing that absorption by dust must be important (Atek et al. 2008).

The other half of the HST sample of Kunth et al. (1998) shows $\operatorname{Ly} \alpha$ profiles in emission and varying amounts of dust

\footnotetext{
2 Two of the objects with Ly $\alpha$ in absorption, IZw 18 and SBS 0335052, have also been observed with FUSE. with a large aperture. The measurements of Grimes et al. (2009) confirm the earlier finding of low velocity shifts, now also on a much larger aperture.
} 
$(E(B-V)$ ranging from $\sim 0.02$ to 0.18$)$. As already mentioned by these authors, the main difference with the other part of the sample showing Ly $\alpha$ absorption appears to be the clear signature of ISM outflows in the former. A continuity of ISM velocities between "static" and "outflowing" is expected and observed (see e.g. Martin 2005; Grimes et al. 2009), mostly correlated with galaxy luminosity, stellar mass, and star formation rate (SFR). A more detailed analysis of the full sample of nearby starbursts observed in Ly $\alpha$ will be presented elsewhere (Atek et al. 2009).

\subsection{Comparison with distant galaxies}

Compared to distant galaxies, it is clear that IZw 18 and SBS 0335-052, or at least the regions of these objects showing intense star formation, show very high HI column density. For example, with $N_{\mathrm{HI}} \sim(0.9-3) \times 10^{21} \mathrm{~cm}^{-2}$, these two regions would correspond to the high $N_{\mathrm{HI}}$ tail of all DLA systems found in the SDSS DR3 survey (cf. Prochaska et al. 2005).

Also, few high- $z$ starbursts with Ly $\alpha$ absorption as broad as in IZw 18 and SBS 0335-052 are known. While $25 \%$ of the LBGs of Shapley et al. (2003) show Ly $\alpha$ absorption, their stacked spectrum shows a narrower absorption profile. Among the broadest Ly $\alpha$ profiles of $z \gtrsim 3$ LBGs are the two lensed galaxies MS 1512-cB58 and FORJ0332-3557, whose absorption profiles corresponds to $\sim(0.7-2.5) \times 10^{21} \mathrm{~cm}^{-2}$ (Pettini et al. 2000; Cabanac et al. 2008).

However, LBGs in general and these two objects in particular differ in many properties compared to IZw 18 . The objects with strong Ly $\alpha$ absorption show significant dust extinction $\left(E_{\star}(B-V) \sim 0.169 \pm 0.006\right.$, where $E_{\star}(B-V)$ is the color excess determined from stellar light), and high SFR (dust-corrected $\left.S F R \sim 52 \pm 5 M_{\odot} \mathrm{yr}^{-1}\right)$. Furthermore outflows with significant velocities $\left(v_{\exp } \sim 100-300 \mathrm{~km} \mathrm{~s}^{-1}\right)$ are generally observed in LBGs. In comparison, IZw 18 is a very low-luminosity, lowSFR object (with a UV luminosity lower than that of LBGs by 2-3 orders of magnitude, $S F R(\mathrm{UV}) \sim 0.3 M_{\odot} \mathrm{yr}^{-1}$ (Grimes et al. $2009)$ with a low extinction $(E(B-V) \lesssim 0.05)$, which shows a static ISM.

For LBGs, Schaerer \& Verhamme (2008) have shown with radiation transfer models that the absorption profile of MS 1512cB58 stems from the relatively large amount of dust and the high column density; with the observed ISM conditions, this suffices to transform intrinsic $\operatorname{Ly} \alpha$ emission expected from the ongoing starburst to broad Ly $\alpha$ absorption, despite the relatively high outflow velocity $\left(v_{\exp } \sim 220 \mathrm{~km} \mathrm{~s}^{-1}\right)$. In IZw 18 geometrical effects or a static high $N_{\mathrm{HI}}$ ISM with small amounts of dust are sufficient to do a similar "transformation".

In short, we suggest schematically the following two explanations for Ly $\alpha$ absorption in nearby and distant starbursts. 1) On average the cold ISM (relevant for Ly $\alpha$ transfer) of LBGs shows the geometry of a spherically expanding shell with relatively high velocities and small variations $\left(v_{\exp } \sim 100-300 \mathrm{~km} \mathrm{~s}^{-1}\right)$ (cf. Shapley et al. 2003; Schaerer \& Verhamme 2008; Verhamme et al. 2008). In such cases the main factors determining the escape fraction of Ly $\alpha$ photons are $N_{\mathrm{HI}}$ and $\tau_{\mathrm{a}}$, as shown by radiation transfer models (Verhamme et al. 2008; Hayes et al. 2009), and significant amounts of dust are required to obtain broad Ly $\alpha$ absorption profiles. 2) In nearby galaxies, small amounts of dust in a static/low velocity ISM with a high HI column density suffice to create Ly $\alpha$ absorption. Furthermore, the occurrence of Ly $\alpha$ absorption is most probably metallicity independent, at least to first order. In addition, geometrical effects due to small apertures may also increase the observed $\operatorname{Ly} \alpha$ absorption.
The distinction between groups 1) and 2) is most likely simply due to the outflow properties, i.e. the wind velocity, which is known to increase with SFR, galaxy mass, and specific SFR (e.g. Martin 2005; Rupke et al. 2005; Schwartz et al. 2006; Grimes et al. 2009). Qualitatively, this increase in the outflow velocity is understood by increasing mechanical feedback on the ISM related to stronger SF activity (SFR) in galaxies with increasing mass or luminosity. At the low-luminosity (SFR) end, feedback appears to be insufficient to "ignite" outflows, hence the nearly static ISM in IZw 18 and alike objects. What ultimately settles the ISM geometry, $N_{\mathrm{HI}}$ and dust-to-gas ratio, and this assures in particular a high HI column density in IZw 18 and other local objects remains to be explained.

Clearly, the observed trends and diversity need to be examined further both qualitatively and quantitatively. This will be the scope of subsequent publications.

\section{Summary and conclusion}

Archival HST/STIS UV spectroscopy and imaging, and HST/WFPC2 optical imaging data of the nearby star-forming galaxy IZw 18, were obtained. We applied the 3D Monte Carlo Ly $\alpha$ radiative transfer code MCLya (Verhamme et al. 2006) to quantitatively explain the intriguing $\operatorname{Ly} \alpha$ absorption in this galaxy and the apparent spatial variation of the Ly $\alpha$ profile. Then, using the example of IZw 18, we discussed under which physical conditions one observes $\operatorname{Ly} \alpha$ in emission or absorption both in nearby or high- $z$ galaxies. Our main results can be summarized as follows.

- We first examined the predictions of a spherical shell model to reproduce the integrated spectrum of the NW region of IZw 18. This model described a static shell of HI mixed with dust grains, surrounding a central point source emitting UV continuum plus a Ly $\alpha$ emission line. Adopting dust extinction derived from observations $(E(B-V)=0.05)$ and $b=20 \mathrm{~km} \mathrm{~s}^{-1}$, we were able to fit the Ly $\alpha$ profile with HI column density of $N_{\mathrm{HI}}=6.5 \times 10^{21} \mathrm{~cm}^{-2}$. Even with a strong intrinsic Ly $\alpha$ emission $E W_{\mathrm{Ly} \alpha}$ (up to $200 \AA$ ), a small amount of dust is enough to cause strong damped Ly $\alpha$ emission, since the probability of absorption by dust is greatly increased by the high column density and by a nearly static ISM. In this model, the loss of Ly $\alpha$ photons is only possible by means of true dust absorption, since we spatially recover all photons.

- Given the large spatial extension of HI covering the NW region (van Zee et al. 1998), we also explored the slab geometry of neutral gas in front of the UV source. We have shown that considering only emission along the observer's line-ofsight can reproduce the strong $\operatorname{Ly} \alpha$ absorption without any dust $(E(B-V)=0)$. This is achieved by the diffusion of the photons out of the observer's sightline provided a sufficiently high column density (at least $N_{\mathrm{HI}}=3 \times 10^{21} \mathrm{~cm}^{-2}$ ) and a nearly static ISM configuration.

- We observed spatial variations in the Ly $\alpha$ profile shape in the different STIS slits. From observations we constructed the $1 \mathrm{D}$ profile variations of the UV continuum and $E W_{\mathrm{Ly} \alpha}$ across the NW region. Despite strong UV emission at the center and high $E W_{\mathrm{Ly} \alpha}$ around, the Ly $\alpha$ profile is still in absorption in all the NW region and proves narrower toward the peripheral region. To understand these variations we simulated an extended source with a spatially varying UV emission strength by following the $1 \mathrm{D}$ spatial profile, in front of a slab of neutral gas with $N_{\mathrm{HI}}=3 \times 10^{21} \mathrm{~cm}^{-2}$ and $E(B-V)=0.05$. Then, 
by observing the slab at different distances from the center, we were able to reproduce the observed spatial variations. We demonstrated qualitatively that this comes from radiative transfer effects, in particular, to the diffusion of Ly $\alpha$ photons and to the spatial variation of the UV continuum source. Finally, adding a Ly $\alpha$ recombination line to the source simulating the observed $E W_{\mathrm{Ly} \alpha}$ profile does not affect the final spectrum.

Other nearby galaxies with intense SF, such as II Zw 70, Mrk 36 and SBS 0335-052, also show strong Ly $\alpha$ absorption (Kunth et al. 1998). They appear to show a nearly static cold ISM and more or less significant amounts of dust. At least two of these objects exhibit very high HI column density (up to $2.4 \times 10^{21} \mathrm{~cm}^{-2}$ ). We suggest that Ly $\alpha$ absorption in these objects stems from the same reasons as for IZw 18: a very large number of scatterings in static, high column density gas leading to an efficient destruction of Ly $\alpha$ photons by even small amounts of dust. Furthermore line-of-sight effects can also be responsible for or increase Ly $\alpha$ absorption.

The distinction between Ly $\alpha$ emission and absorption in local starbursts seems to be mainly related to the presence or not of ISM outflows. Since high- $z$ objects (LBGs, LAEs) generally show outflows with high, but relatively similar velocities (with bulk velocity typically $\sim 100-200 \mathrm{~km} \mathrm{~s}^{-1}$ ), the transition from Ly $\alpha$ absorption to emission in these objects is, on the other hand, mostly determined by the dust content and HI column density (cf. Schaerer \& Verhamme 2008; Verhamme et al. 2008).

These results and the global trends observed between Ly $\alpha$ strength and profile diversity, and their relevant parameters now need to be tested with larger samples of galaxies. This is the main objective of upcoming publications (e.g. Atek et al. 2009).

Acknowledgements. We would like to warmly thank Anne Verhamme, Matthew Hayes, Sally Heap, and Françoise Combes for useful discussions. We are also grateful to Miguel Mas-Hesse for providing us with his UV spectra and for useful comments. Simulations were done on the regor PC cluster at the Geneva Observatory co-funded by grants to Georges Meynet, Daniel Pfenniger, and DS. H.A. and D.K. are supported by the Centre National d'Études Spatiales (CNES). The work of D.S. is supported by the Swiss National Science Foundation.

\section{References}

Ajiki, M., Taniguchi, Y., Fujita, S. S., et al. 2003, AJ, 126, 2091

Atek, H., Kunth, D., Hayes, M., Östlin, G., \& Mas-Hesse, J. M. 2008, A\&A, 488,491

Atek, H., Kunth, D., Schaerer, D., \& Deharveng, J. M. 2009, A\&A, in preparation

Bravo-Alfaro, H., Brinks, E., Baker, A. J., Walter, F., \& Kunth, D. 2004, AJ, 127, 264

Brocklehurst, M. 1971, MNRAS, 153, 471

Brown, T. M., Heap, S. R., Hubeny, I., Lanz, T., \& Lindler, D. 2002, ApJ, 579, L75

Cabanac, R. A., Valls-Gabaud, D., \& Lidman, C. 2008, MNRAS, 386, 2065

Cannon, J. M., Skillman, E. D., Garnett, D. R., \& Dufour, R. J. 2002, ApJ, 565, 931

Cardelli, J. A., Clayton, G. C., \& Mathis, J. S. 1989, ApJ, 345, 245

Charlot, S., \& Fall, S. M. 1993, ApJ, 415, 580

de Mello, D. F., Schaerer, D., Heldmann, J., \& Leitherer, C. 1998, ApJ, 507, 199

Deharveng, J. M., Joubert, M., \& Kunth, D. 1985, in Star-Forming Dwarf Galaxies and Related Objects, ed. D. Kunth, T. X. Thuan, \& J. Tran Thanh van, 431

Deharveng, J.-M., Small, T., Barlow, T. A., et al. 2008, ApJ, 680, 1072

Dijkstra, M., \& Loeb, A. 2008, MNRAS, 386, 492

Dijkstra, M., Haiman, Z., \& Spaans, M. 2006, ApJ, 649, 14

Dufour, R. J., Garnett, D. R., \& Shields, G. A. 1988, ApJ, 332, 752
Engelbracht, C. W., Rieke, G. H., Gordon, K. D., et al. 2008, ApJ, 678, 804

Giavalisco, M., Koratkar, A., \& Calzetti, D. 1996, ApJ, 466, 831

Grimes, J. P., Heckman, T., Aloisi, A., et al. 2009, ApJS, 181, 272

Gronwall, C., Ciardullo, R., Hickey, T., et al. 2007, ApJ, 667, 79

Hansen, M., \& Peng Oh, S. 2006, New Astron. Rev., 50, 58

Hartmann, L. W., Huchra, J. P., \& Geller, M. J. 1984, ApJ, 287, 487

Hartmann, L. W., Huchra, J. P., Geller, M. J., O’Brien, P., \& Wilson, R. 1988, ApJ, 326, 101

Hayes, M., Östlin, G., Mas-Hesse, J. M., et al. 2005, A\&A, 438, 71

Hayes, M., Östlin, G., Mas-Hesse, J. M., \& Kunth, D. 2008, AJ, submitted

Hayes, M., Schaerer, D., \& Verhamme, A. 2009, A\&A, in preparation

Houck, J. R., Charmandaris, V., Brandl, B. R., et al. 2004, ApJS, 154, 211

Hu, E. M., Cowie, L. L., \& McMahon, R. G. 1998, ApJ, 502, L99

Hunter, D. A., \& Thronson, Jr., H. A. 1995, ApJ, 452, 238

Izotov, Y. I., \& Thuan, T. X. 1998, ApJ, 500, 188

Izotov, Y. I., \& Thuan, T. X. 1999, ApJ, 511, 639

Izotov, Y. I., Foltz, C. B., Green, R. F., Guseva, N. G., \& Thuan, T. X. 1997, ApJ, 487, L37

Kashikawa, N., Shimasaku, K., Malkan, M. A., et al. 2006, ApJ, 648, 7

Kudritzki, R.-P., Méndez, R. H., Feldmeier, J. J., et al. 2000, ApJ, 536, 19

Kunth, D., Lequeux, J., Sargent, W. L. W., \& Viallefond, F. 1994, A\&A, 282, 709

Kunth, D., Mas-Hesse, J. M., Terlevich, E., et al. 1998, A\&A, 334, 11

Kunth, D., Leitherer, C., Mas-Hesse, J. M., Östlin, G., \& Petrosian, A. 2003, ApJ, 597, 263

Lequeux, J., Kunth, D., Mas-Hesse, J. M., \& Sargent, W. L. W. 1995, A\&A, 301, 18

Malhotra, S., \& Rhoads, J. E. 2002, ApJ, 565, L71

Martin, C. L. 1996, ApJ, 465, 680

Martin, C. L. 2005, ApJ, 621, 227

Mas-Hesse, J. M. 1990, Galaxias con formacion ESTELAR violenta, Madrid: Universidad

Mas-Hesse, J. M., Kunth, D., Tenorio-Tagle, G., et al. 2003, ApJ, 598, 858

Meier, D. L., \& Terlevich, R. 1981, ApJ, 246, L109

Neufeld, D. A. 1990, ApJ, 350, 216

Neufeld, D. A. 1991, ApJ, 370, L85

Nilsson, K. K., Tapken, C., Møller, P., et al. 2009, A\&A, 498, 13

Ouchi, M., Shimasaku, K., Akiyama, M., et al. 2008, ApJS, 176, 301

Partridge, R. B., \& Peebles, P. J. E. 1967, ApJ, 147, 868

Péquignot, D. 2008, A\&A, 478, 371

Pettini, M., Steidel, C. C., Adelberger, K. L., Dickinson, M., \& Giavalisco, M. 2000, ApJ, 528, 96

Prochaska, J. X., Herbert-Fort, S., \& Wolfe, A. M. 2005, ApJ, 635, 123

Pustilnik, S. A., Brinks, E., Thuan, T. X., Lipovetsky, V. A., \& Izotov, Y. I. 2001, AJ, 121, 1413

Rupke, D. S., Veilleux, S., \& Sanders, D. B. 2005, ApJS, 160, 115

Schaerer, D. 2003, A\&A, 397, 527

Schaerer, D. 2007, ArXiv e-prints

Schaerer, D., \& Verhamme, A. 2008, A\&A, 480, 369

Schlegel, D. J., Finkbeiner, D. P., \& Davis, M. 1998, ApJ, 500, 525

Schwartz, C. M., Martin, C. L., Chandar, R., et al. 2006, ApJ, 646, 858

Shapley, A. E., Steidel, C. C., Pettini, M., \& Adelberger, K. L. 2003, ApJ, 588, 65

Shimasaku, K., Kashikawa, N., Doi, M., et al. 2006, PASJ, 58, 313

Skillman, E. D., \& Kennicutt, Jr., R. C. 1993, ApJ, 411, 655

Stenflo, J. O. 1980, A\&A, 84, 68

Taniguchi, Y., Ajiki, M., Nagao, T., et al. 2005, PASJ, 57, 165

Tapken, C., Appenzeller, I., Gabasch, A., et al. 2006, A\&A, 455, 145

Tenorio-Tagle, G., Silich, S. A., Kunth, D., Terlevich, E., \& Terlevich, R. 1999, MNRAS, 309, 332

Terlevich, E., Diaz, A. I., Terlevich, R., \& Vargas, M. L. G. 1993, MNRAS, 260, 3

Thuan, T. X., \& Izotov, Y. I. 1997, ApJ, 489, 623

Thuan, T. X., Izotov, Y. I., \& Lipovetsky, V. A. 1997, ApJ, 477, 661

Thuan, T. X., Sauvage, M., \& Madden, S. 1999, ApJ, 516, 783

Valls-Gabaud, D. 1993, ApJ, 419, 7

van Zee, L., Westpfahl, D., Haynes, M. P., \& Salzer, J. J. 1998, AJ, 115, 1000

Verhamme, A., Schaerer, D., \& Maselli, A. 2006, A\&A, 460, 397

Verhamme, A., Schaerer, D., Atek, H., \& Tapken, C. 2008, A\&A, 491, 89

Vílchez, J. M., \& Iglesias-Páramo, J. 1998, ApJ, 508, 248

Witt, A. N., \& Gordon, K. D. 2000, ApJ, 528, 799

Wu, Y., Charmandaris, V., Hunt, L. K., et al. 2007, ApJ, 662, 952

Zheng, Z., \& Miralda-Escudé, J. 2002, ApJ, 578, 33

Zwicky, F. 1966, ApJ, 143, 192 